\title{
Livestock Rearing Contribution towards the Beneficiary and non- Beneficiary on Watershed Development Programme in Nagaland
}

\author{
Mukesh Kumar Yadav and Amod Sharma* \\ Department of Agricultural Economics, Nagaland University SASRD Medziphema Campus, \\ District: Dimapur - 797 106, Nagaland, India \\ *Corresponding author
}

Keywords

Nagaland, income, employment, beneficiaries, nonbeneficiaries, activities.

Article Info

Accepted:

25 August 2019

Available Online:

10 September 2019
A B S T R A C T

The present study to access the assured income and employment through different activities implemented under watershed programme with reference to the beneficiaries and non-beneficiaries for the purposely selected two districts from the Nagaland state viz; Dimapur and Kohima as both were selected purposely due to the maximum number of area covered under watershed in the zone and further two blocks from each district were randomly selected, while in the second stage a multi stage random purposive sampling methods viz; 320 respondents (160 beneficiaries and 160 non-beneficiaries) were selected randomly from identified watershed areas. Study reveals that the average annual or monthly income of the beneficiary always be more than the non-beneficiary. Even on beneficiary it increase with increasing in the farm size group, respectively in the rural areas of Nagaland.

\section{Introduction}

Watershed management activities is the process of guiding and organizing land, soil and other resource use on a watershed to provide needed goods and services and simultaneously conserving soil, water and land natural resources. The interrelationships among soil land used and water, and the linkages between up-stream and downstream area are given an explicit significance in watershed approach. Watershed management focuses on using resources in a productive and sustainable manner. The primary objective of watershed management is to slow down or if possible reversing the manmade degradation which is mostly manifested in accelerated runoff usually with heavy sedimentations, reduced agricultural productivity and progressive removal of vegetative cover on non-arable land. watershed management project help in internalizing the externalities 
caused by flooding from a large number of seasonal torrents every year. Since 1970, there have been heavy investments by Central and State Governments in the watershed development projects. Integrated Watershed Management (IWM) has been identified as a key for planning and management of natural resources in mountain ecosystems. It provides an ecologically sound economic base for the watersheds and its people. In any developmental activity, the watershed approach is more scientific because the inherent potential of soil, water and forest recourses in a particular area is controlled by various factors such as physiography, geological base, soil characteristic, climate, present land use, socio-economic aspects etc. ((Anon. 2016)).

Kohima, the capital of Nagaland, is a hilly district sharing its borders with Dimapur in the West, Phek District in the East, Peren in the South and Wokha in the North. It has a humid subtropical climate, with an elevation of 1444 metres and covers an area of 1463 sq. km. While the Dimapur District is the centre for many commercial activities. It is bounded by Kohima district on the South and East, KarbiAnglong on the West, Golaghat District of Assam, in the North. A large area of the District is in the plains with an average elevation of $260 \mathrm{~m}$ above sea level with an area of 927 sq. km (Anon. 2017).

\section{Materials and Methods}

The present study is related to IWMP scheme, which is working as per the guideline of Central government with the help of Ministry of Agriculture, Government of India. Development projects require long period of time to reap benefits. Therefore for economic appraisal of development projects, it is essential that the project has been in operation for quite some time. Since the intensive IWMP started in 2008-09, so it is worth, while to study its impact. Since the data of the initial period cannot be compared with the data of recent years. It is more scientific and practical to compare the economy of the beneficiaries and non-beneficiaries covered in the area of IWMP schemes.

The IWMP was launched in 2008-09 in all 11 district viz: Dimapur, Kohima, Kiphire, Longleng, Mokokchung, Mon, Phek, Peren, Tuensang, Wokha and Zunheboto of Nagaland, out of these districts two districts namely, Zunheboto and Dimapur districts of Nagaland selected because of the fact that it is expected to provide all the relevant information and hence can conveniently be obtained for conducting this study. (Anon., 2017). The project area also has a good network of infrastructure and allied activities related to the scheme such as development agencies, nationalized banks, well-established marketing and communication facilities etc.

Keeping all the above facts, both districts of Nagaland are therefore purposively selected, while two blocks were selected from the selected districts, while from each blocks 2 villages ( 8 villages in total) were selected randomly and then 10 beneficiaries and 10 non-beneficiaries (20 cases in total from each villages), so 80 numbers each of beneficiaries and non-beneficiaries were selected by following the simple systematic randomly sampling techniques with the specific objectives to conduct the present study viz; to examine the income contribution through livestock enterprises and its activities adopted under watershed management programme.

\section{Results and Discussion}

Table 1 reveals the overall / average total cost investment of animal on beneficiaries, which was found to be less (Rs 27187.37) as compare to the non-beneficiaries (Rs 27581.25), even the overall average total cost / 
cost of cultivation of animals on beneficiaries was recorded as maximum Rs 9453.12 (34.77 per cent) on medication charges, followed by transportation cost with Rs 6640.62 (24.43 per cent), feeding cost was Rs 4861.87 (17.88 per cent), cost of animal shed was Rs 2431.06 (8.94 per cent), other charges was of Rs 1460.87 (5.37 per cent), Rs 1186.87 (4.36 per cent) on labour cost and it was found to be least on equipment as Rs 1152.94 (4.24 per cent), respectively. Similar studies were find out by the Sharma (2002); Sharma and Sharma (2008); Shuya and Sharma (2014); Walling and Sharma (2015); Walling et al., (2017); Shuya and Sharma (2018).

Further table reveals that the overall / average total cost investment of animal on nonbeneficiaries was recorded as maximum Rs 8669.69 (31.43 per cent) on feeding cost, followed by Rs 5279.37 (19.14 per cent) on medication charges, cost of animal shed with Rs 4212.50 (15.27 per cent), transportation cost was Rs 3989.37 (14.46 per cent), labour cost was Rs 3876.87 (14.06 per cent), Rs 1016.56 (3.69 per cent) for other charges and Rs 536.87 (1.95 per cent) on the equipment as least cost item, respectively. Similar studies were find out by the Sharma (2004); Sharma (2011); Mishra et al., (2014); Sharma et al., (2016); Walling et al., (2017); Sangtam and Sharma (2015); Pongeneer and Sharma (2018).

Table 2 reveals the farm size as well as average farm inventory status of livestock animals on beneficiaries, which was found to be more (Rs 31136.64) as compare to the nonbeneficiaries (Rs 29255.62), while the overall total assets of animals on beneficiaries was recorded as maximum Rs 22363.75 (71.82 per cent) of piggery reared in cost, followed by cattle numbers as reared cost with Rs 5612.50 (18.02 per cent), poultry reared cost was Rs 1711.88 (5.81 per cent), while on other animals reared cost will be found to be least as Rs 1431.25 (4.59 per cent), respectively. Similar studies were find out by the Sharma (2002); Sharma and Sharma (2008); Shuya and Sharma (2014); Walling and Sharma (2015); Walling et al., (2017); Shuya and Sharma (2018).

Further table reveals that the overall total assets of animals on non-beneficiaries was recorded as maximum Rs 22368.75 (76.46 per cent) of piggery reared in cost, followed by cattle numbers as reared cost with Rs 5321.88 (18.19 per cent), poultry reared cost was Rs 1000.00 (3.18 per cent), while on other animals reared cost will be found to be least as Rs 565.00 (1.93 per cent), respectively. Similar studies were find out by the Sharma (2004); Sharma (2011); Mishra et al., (2014); Sharma et al., (2016); Walling et al., (2017); Sangtam and Sharma (2015); Pongeneer and Sharma (2018).

Table 3 reveals the overall average return from different livestock enterprises on beneficiaries, which was found to be more (Rs 56938.36) as compare to the non-beneficiaries (Rs 34021.87), respectively. While the overall total return through livestock on beneficiaries was recorded as maximum on Piggery farm Rs 38492.50 (67.60 per cent), followed by cattle with Rs 14003.36 (24.59 per cent), through poultry rearing return was Rs 2636.25 (4.63 per cent), while it was found least by the other reared returns as Rs 1806.25 (3.18 per cent), respectively.

While among the farm size group the net return has been increased with increased in the farm size group. Similar studies were find out by the Sharma (2002); Sharma and Sharma (2008); Shuya and Sharma (2014); Walling and Sharma et al., (2015); Walling et al., (2017); Shuya and Sharma (2018). 
Table.1 Cost of animals of beneficiaries and non-beneficiaries respondent families

\begin{tabular}{|c|c|c|c|c|c|c|c|c|c|}
\hline \multicolumn{2}{|r|}{ Groups } & Cost of & Feeding cost & Medication & Equipments & Labour & Transportation & Others & Total \\
\hline \multirow{8}{*}{ 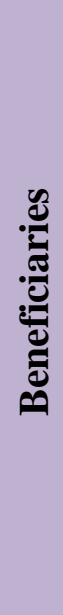 } & \multirow[t]{2}{*}{ Small } & 2186.84 & 4794.74 & 8705.26 & 1068.42 & 1089.47 & 6005.26 & 1489.47 & 25339.47 \\
\hline & & (8.63) & (18.92) & $(34.35)$ & $(4.22)$ & $(4.30)$ & $(23.70)$ & $(5.88)$ & (100.00) \\
\hline & \multirow[t]{2}{*}{ Medium } & 2353.12 & 5281.25 & 9775 & 1052.5 & 1150 & 6031.25 & 1445.31 & 27088.43 \\
\hline & & (8.69) & (19.50) & $(36.08)$ & (3.88) & $(4.24)$ & $(22.27)$ & $(5.34)$ & (100.00) \\
\hline & \multirow[t]{2}{*}{ Large } & 2496.51 & 4750.46 & 9488.99 & 1197.16 & 1214.68 & 6930.27 & 1460.45 & 27538.52 \\
\hline & & (9.07) & $(17.25)$ & $(34.46)$ & $(4.35)$ & $(4.41)$ & $(25.17)$ & $(5.30)$ & (100.00) \\
\hline & \multirow[t]{2}{*}{ Average } & 2431.06 & 4861.87 & 9453.12 & 1152.94 & 1186.87 & 6640.62 & 1460.87 & 27187.37 \\
\hline & & (8.94) & (17.88) & (34.77) & (4.24) & $(4.36)$ & (24.43) & $(5.37)$ & (100.00) \\
\hline \multirow{8}{*}{ 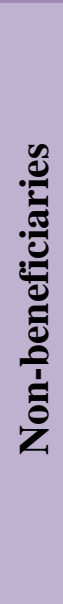 } & \multirow[t]{2}{*}{ Small } & 3020.83 & 5454.17 & 8625 & 566.67 & 1466.67 & 5816.67 & 916.67 & 25866.67 \\
\hline & & (11.68) & (21.09) & (33.34) & (2.19) & $(5.67)$ & $(22.49)$ & $(3.54)$ & (100.00) \\
\hline & \multirow[t]{2}{*}{ Medium } & 3972.50 & 8105.62 & 5535 & 522.5 & 3567.5 & 4255 & 1008.75 & 26966.87 \\
\hline & & (14.73) & $(30.06)$ & (20.53) & (1.94) & $(13.23)$ & (15.78) & $(3.74)$ & $(100.00)$ \\
\hline & \multirow[t]{2}{*}{ Large } & 4705.15 & 9900.73 & 4388.23 & 548.53 & 4666.17 & 3354.41 & 1043.38 & 28606.62 \\
\hline & & $(16.45)$ & $(34.61)$ & $(15.34)$ & $(1.92)$ & $(16.31)$ & (11.73) & $(3.65)$ & $(100.00)$ \\
\hline & \multirow[t]{2}{*}{ Average } & 4212.5 & 8669.69 & 5279.37 & 536.87 & 3876.87 & 3989.37 & 1016.56 & 27581.25 \\
\hline & & (15.27) & $(31.43)$ & (19.14) & (1.95) & (14.06) & (14.46) & $(3.69)$ & (100.00) \\
\hline
\end{tabular}

(The figure in the parentheses indicates percentage in total) 
Table.2 Animal inventories status of beneficiaries and non-beneficiaries

\begin{tabular}{|c|c|c|c|c|c|c|c|c|c|c|}
\hline \multicolumn{2}{|r|}{ Groups } & \multicolumn{2}{|c|}{ Cattle } & \multicolumn{2}{|c|}{ Poultry } & \multicolumn{2}{|c|}{ Piggery } & \multicolumn{2}{|c|}{ Others } & \multirow[t]{2}{*}{ Total value } \\
\hline & & $\begin{array}{l}\text { Nos. } \\
\text { reared }\end{array}$ & $\begin{array}{c}\text { Present } \\
\text { value }\end{array}$ & $\begin{array}{l}\text { Nos. } \\
\text { reared }\end{array}$ & $\begin{array}{c}\text { Present } \\
\text { value }\end{array}$ & $\begin{array}{c}\text { Nos. } \\
\text { reared }\end{array}$ & $\begin{array}{c}\text { Present } \\
\text { value }\end{array}$ & $\begin{array}{c}\text { Nos. } \\
\text { reared }\end{array}$ & $\begin{array}{c}\text { Present } \\
\text { value }\end{array}$ & \\
\hline \multirow{8}{*}{ 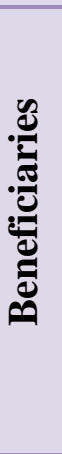 } & \multirow[t]{2}{*}{ Small } & 17 & 17421.05 & 55 & 100 & 11 & 32000 & 120 & 1263.16 & 50784.21 \\
\hline & & $(0.03)$ & $(34.3)$ & $(0.10)$ & $(0.10)$ & $(0.02)$ & $(63.01)$ & $(0.24)$ & $(2.48)$ & $(100.00)$ \\
\hline & \multirow[t]{2}{*}{ Medium } & 8 & 4187.5 & 180 & 1125 & 24 & 15312.5 & 480 & 3031.25 & 24348.25 \\
\hline & & $(0.03)$ & $(17.19)$ & $(0.74)$ & $(4.63)$ & $(0.09)$ & $(62.89)$ & $(1.97)$ & $(12.46)$ & $(100.00)$ \\
\hline & \multirow[t]{2}{*}{ Large } & 23 & 3972.48 & 1180 & 2165.14 & 125 & 22754.13 & 540 & 990.83 & 31750.58 \\
\hline & & $(0.07)$ & $(12.51)$ & $(3.72)$ & $(6.82)$ & $(0.40)$ & $(71.66)$ & $(1.70)$ & $(3.12)$ & $(100.00)$ \\
\hline & \multirow[t]{2}{*}{ Average } & 0.3 & 5612.5 & 8.84 & 1711.88 & 1.00 & 22363.75 & 7.125 & 1431.25 & 31136.64 \\
\hline & & $(0.00)$ & (18.02) & $(0.03)$ & $(5.49)$ & $(0.00)$ & (71.82) & $(0.02)$ & $(4.59)$ & $(100.00)$ \\
\hline \multirow{8}{*}{ 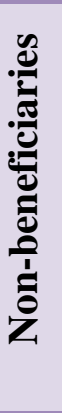 } & \multirow[t]{2}{*}{ Small } & 8 & 3333.33 & 130 & 1833.33 & 52 & 24916.67 & 0 & 0 & 30083.33 \\
\hline & & $(0.02)$ & (11.08) & $(0.43)$ & $(6.09)$ & $(0.17)$ & $(82.82)$ & $(0.00)$ & $(0.00)$ & $(100.00)$ \\
\hline & \multirow[t]{2}{*}{ Medium } & 49 & 6043.75 & 410 & 862.5 & 358 & 236000 & 720 & 292.5 & 243198.75 \\
\hline & & $(0.02)$ & $(2.48)$ & $(0.17)$ & $(0.34)$ & $(0.14)$ & $(97.04)$ & $(0.29)$ & $(0.12)$ & $(100.00)$ \\
\hline & \multirow[t]{2}{*}{ Large } & 34 & 4823.53 & 390 & 1014.71 & 255 & 20470.58 & 400 & 985.29 & 27294.11 \\
\hline & & $(0.12)$ & $(17.67)$ & $(1.43)$ & $(3.72)$ & $(0.93)$ & $(74.99)$ & $(1.46)$ & $(3.6)$ & $(100.00)$ \\
\hline & \multirow[t]{2}{*}{ Average } & 0.57 & 5321.88 & 5.81 & 1000.0 & 4.16 & 22368.75 & 7.0 & 565.00 & 29255.62 \\
\hline & & $(0.00)$ & (18.19) & $(0.019)$ & (3.18) & $(0.014)$ & $(76.46)$ & $(0.023)$ & (1.93) & $(100.00)$ \\
\hline
\end{tabular}

(The figure in the parentheses indicates percentage in total) 
Table.3 Return from different animal husbandry activity

\begin{tabular}{|c|c|c|c|c|c|c|}
\hline \multirow{2}{*}{\multicolumn{2}{|c|}{ Groups }} & \multicolumn{5}{|c|}{ Return from } \\
\hline & & Cattle & Poultry & Piggery & Others & Total return \\
\hline \multirow{8}{*}{ } & \multirow[t]{2}{*}{ Small } & 9316.53 & 894.74 & 13936.84 & 1552.63 & 25700.74 \\
\hline & & $(36.25)$ & $(3.48)$ & $(54.23)$ & $(6.04)$ & $(\mathbf{1 0 0 . 0 0 )}$ \\
\hline & \multirow{2}{*}{ Medium } & 8422 & 2053.12 & 35231.25 & 1621.88 & 47327.25 \\
\hline & & $(17.79)$ & $(4.34)$ & $(74.44)$ & $(3.43)$ & $(100.00)$ \\
\hline & \multirow[t]{2}{*}{ Large } & 7541.47 & 3111.01 & 43730.27 & 611.01 & 54993.76 \\
\hline & & $(13.71)$ & $(5.66)$ & $(79.52)$ & (1.11) & $(\mathbf{1 0 0 . 0 0 )}$ \\
\hline & \multirow[t]{2}{*}{ Average } & 14003.36 & 2636.25 & 38492.5 & 1806.25 & 56938.36 \\
\hline & & $(24.59)$ & $(4.63)$ & $(67.60)$ & $(3.18)$ & $(\mathbf{1 0 0 . 0 0 )}$ \\
\hline \multirow{8}{*}{ 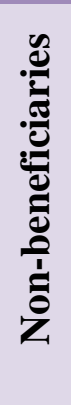 } & \multirow[t]{2}{*}{ Small } & 6625.0 & 2016.67 & 25183.33 & 0.00 & 33825.00 \\
\hline & & (19.59) & $(5.96)$ & $(74.45)$ & $(0.00)$ & $(\mathbf{1 0 0 . 0 0 )}$ \\
\hline & \multirow[t]{2}{*}{ Medium } & 10243.75 & 965.0 & 23822.5 & 1507.5 & 36538.75 \\
\hline & & (28.04) & (2.64) & $(6.52)$ & $(4.13)$ & $(\mathbf{1 0 0 . 0 0 )}$ \\
\hline & \multirow[t]{2}{*}{ Large } & 8323.53 & 1123.35 & 20654.41 & 985.29 & 31095.59 \\
\hline & & (26.77) & (3.61) & $(66.42)$ & (3.17) & $(100.00)$ \\
\hline & \multirow[t]{2}{*}{ Average } & 9156.25 & 1115.0 & 22578.12 & 1172.5 & 34021.87 \\
\hline & & (26.91) & (3.28) & (66.36) & (3.45) & $(100.00)$ \\
\hline
\end{tabular}

(The figure in the parentheses indicates percentage in total)

Fig.1 Distribution of sampled respondent family groups of beneficiaries and non-beneficiaries according to cost of animal production

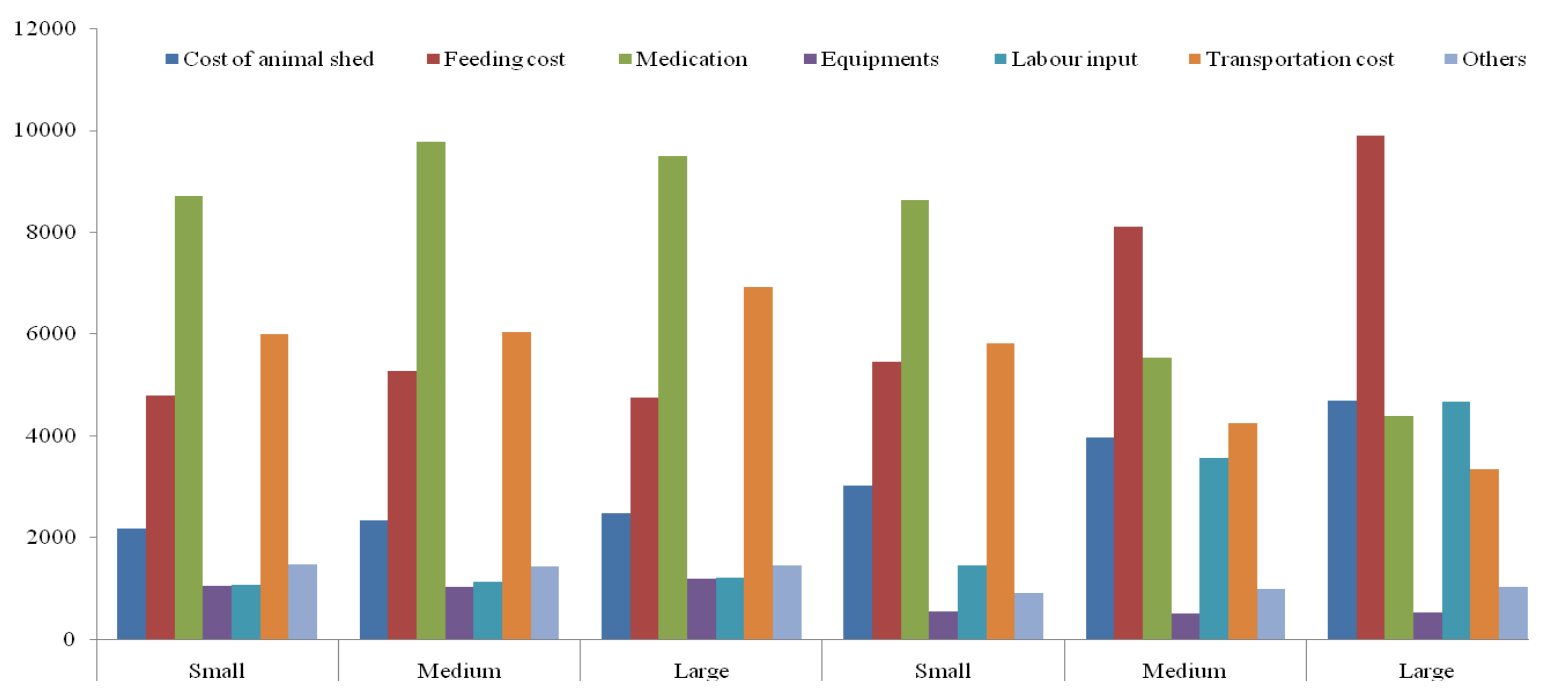


Table.4 Item wise return from different animal husbandry activity of beneficiaries and non-beneficiaries respondents

\begin{tabular}{|c|c|c|c|c|c|c|c|c|c|c|}
\hline \multirow{2}{*}{\multicolumn{2}{|c|}{ Groups }} & \multicolumn{2}{|c|}{ Young } & \multicolumn{2}{|c|}{ Mature } & \multirow{3}{*}{$\begin{array}{c}\text { Milk } \\
2973.68\end{array}$} & \multirow{3}{*}{$\begin{array}{c}\text { Egg } \\
148.95\end{array}$} & \multirow{3}{*}{$\begin{array}{l}\text { Manure } \\
2673.68\end{array}$} & \multirow{3}{*}{$\begin{array}{l}\text { Others } \\
852.63\end{array}$} & \multirow{3}{*}{$\begin{array}{c}\text { Total } \\
41543.67 \\
\end{array}$} \\
\hline & & Nos. & Value & Nos. & Value & & & & & \\
\hline \multirow{8}{*}{ 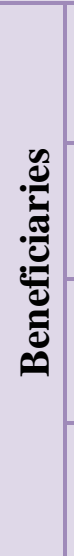 } & \multirow[t]{2}{*}{ Small } & 5.52 & 13421.05 & 10.58 & 21473.68 & & & & & \\
\hline & & $(0.015)$ & (15.96) & $(0.03)$ & (58.67) & $(16.32)$ & $(0.22)$ & (7.30) & (1.51) & $(100.00)$ \\
\hline & \multirow[t]{2}{*}{ Medium } & 11.47 & 17296.87 & 19.44 & 29343.75 & 3656.25 & 154.65 & 1953.12 & 1621.87 & 54026.51 \\
\hline & & $(0.02)$ & $(28.17)$ & $(0.04)$ & $(62.16)$ & $(3.50)$ & $(0.24)$ & $(2.44)$ & $(3.43)$ & $(100.00)$ \\
\hline & \multirow[t]{2}{*}{ Large } & 13.28 & 18399.08 & 18.84 & 32973.78 & 3976.15 & 179.63 & 2813.76 & 1784.01 & 60126.41 \\
\hline & & $(0.02)$ & (33.33) & $(0.03)$ & $(59.68)$ & (3.39) & $(0.29)$ & $(2.19)$ & (1.11) & $(100.00)$ \\
\hline & \multirow[t]{2}{*}{ Average } & 12.00 & 16372.67 & 17.98 & 30882.14 & 3602.03 & 161.08 & 2480.19 & 1482.17 & 54998.26 \\
\hline & & $(0.02)$ & $(30.89)$ & $(\mathbf{0 . 0 3})$ & $(60.05)$ & $(4.51)$ & $(0.27)$ & (2.67) & (1.57) & $(100.00)$ \\
\hline \multirow{8}{*}{ 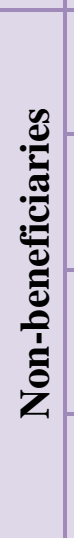 } & \multirow[t]{2}{*}{ Small } & 6.42 & 9166.67 & 9.42 & 20916.67 & 2250.00 & 100.00 & 1391.67 & 0.00 & 33825.0 \\
\hline & & $(0.02)$ & $(27.10)$ & $(0.03)$ & (61.84) & $(6.65)$ & $(0.29)$ & $(4.11)$ & $(0.00)$ & $(100.00)$ \\
\hline & \multirow[t]{2}{*}{ Medium } & 5.26 & 10510.00 & 4.95 & 20356.25 & 3162.50 & 52.50 & 1310.00 & 1507.50 & 36538.75 \\
\hline & & $(0.01)$ & $(28.76)$ & $(0.01)$ & $(55.71)$ & $(8.65)$ & $(0.14)$ & $(3.58)$ & $(4.12)$ & $(\mathbf{1 0 0 . 0 0})$ \\
\hline & \multirow[t]{2}{*}{ Large } & 4.82 & 7860.29 & 5.16 & 18448.53 & 2720.59 & 58.82 & 1022.06 & 985.29 & 31095.59 \\
\hline & & $(0.02)$ & $(25.28)$ & $(0.02)$ & (59.33) & $(8.75)$ & $(0.19)$ & $(3.29)$ & $(3.17)$ & (100.00) \\
\hline & \multirow[t]{2}{*}{ Average } & 5.16 & 9103.12 & 5.37 & 19587.5 & 2906.25 & 58.75 & 1193.75 & 1172.5 & 34021.87 \\
\hline & & $(0.01)$ & $(26.76)$ & $(0.01)$ & (57.57) & $(8.54)$ & $(0.17)$ & $(3.51)$ & $(3.45)$ & $(\mathbf{1 0 0 . 0 0 )}$ \\
\hline
\end{tabular}


Fig.2 Distribution of sampled respondent family groups animal husbandry

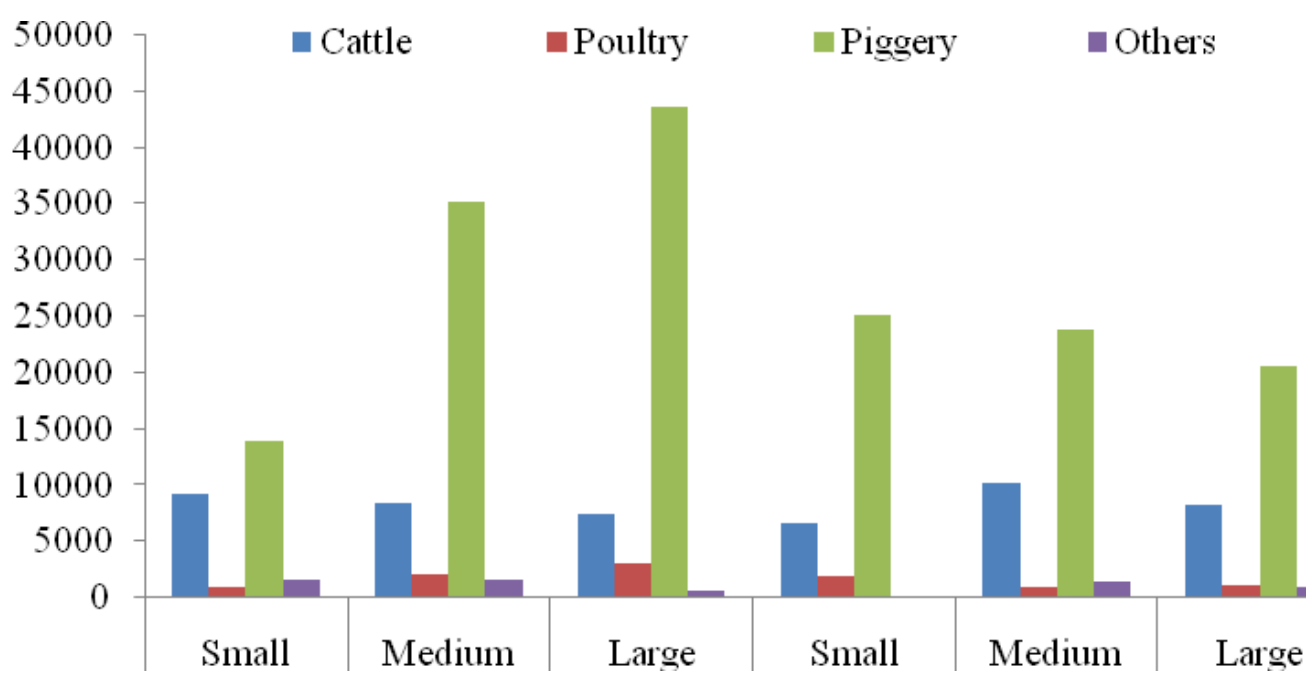

Further the overall total return through livestock on non-beneficiaries was recorded as maximum on Piggery farm with Rs 22578.12 (66.36 per cent), followed by cattle with Rs 9156.25 (26.91 per cent), through other animals reared return was Rs 1172.50 (3.45 per cent), while it was found least by the poultry rearing return as Rs 1115.00 (3.28 per cent), respectively. While among the farm size group the net return was found to be maximum on medium farm size group, followed by marginal and it was found to be minimum on large farm size group, respectively. Similar studies were find out by the Sharma (2004); Sharma and Sharma (2008); Dhakre and Sharma (2010); Sharma (2012); Shuya and Sharma (2014); Sharma (2014); Sangtam and Sharma (2015); Walling and Sharma, (2015); Sharma et al., (2018).

Table 4 reveals the overall average return from item wise different livestock enterprises on beneficiaries, which was found to be more (Rs 54998.26) as compare to the nonbeneficiaries (Rs 34021.87), respectively. While the overall total return through livestock on beneficiaries was recorded as maximum on Mature animals was Rs
30882.34 (60.05 per cent), followed by cattle with Rs 16372.67 (30.89 per cent), through milk the return was Rs 3602.03 (4.51 per cent), manure contribute Rs 2480.19 (2.67 per cent), through others it was Rs 1482.17 (1.57 per cent), while it was found least as egg return investment towards the farm as Rs 161.08 (0.27 per cent), respectively.

Whereas, among the farm size group the net return has been increased with increased in the farm size group. Similar studies were find out by the Sharma (2002); Sharma and Sharma (2008); Shuya and Sharma (2014); Walling and Sharma (2015); Walling et al., (2017).

Further the overall total return through livestock on beneficiaries was recorded as maximum on Mature animals was Rs 19587.50 (57.57 per cent), followed by cattle with Rs 9103.12 (26.76 per cent), through milk the return was Rs 2906.25 (8.54 per cent), manure contribute Rs 1193.75 (3.51 per cent), through others it was Rs 1172.50 (3.45 per cent), while it was found least as egg return investment towards the farm as Rs 58.75 ( 0.17 per cent), respectively. Whereas, among the farm size group the net return has 
been increased with increased in the farm size group. Similar studies were find out by the Sharma (2002); Sharma (2011); Mishra et al., (2014); Sharma et al., (2016); Sangtam and Sharma (2015); Walling et al., (2017); Pongeneer and Sharma (2018); Tangjang and Sharma (2018).

\section{References}

Analogous. 2016. Agricultural Situation in India. Directorate of Economics and Statistics. Ministry of Agriculture, New Delhi.

Analogous. 2017. Statistical Hand of Nagaland Published by Directorate of Economics and Statistics (various issues), Kohima, Nagaland.

Dhakre, D. S. and Sharma, Amod. 2010. Socio-Economic Development in India. Environment and Ecology. 4(1): 2469-2472.

Mishra, A.; Pattnaik, B. R. and Ray, Plabita. 2014. Impact of Watershed Development Programme on Socioeconomic Development of the People. Journal of Extension Education. 39(1): 182-189.

Pongener, Bendangjungla. and Sharma, Amod. 2018. Constraints Faced by the Fishery Enterprises: A SWOC Analysis. IJCMAS. 7(5). May: 15951603.

Sangtam, Likhase. L. T. and Sharma, Amod. 2015. Impact of Bank Finance on Employment and Income through Piggery Enterprise in Nagaland. EPRAIJEBR. 3(11). Nov: 273-276.

Sharma, A. 2002. Source and Knowledge on beneficiaries about the purpose of credit - A case study of Agra Region of Uttar Pradesh. Journal of Interacademica. 6(3). July: 374-379.

Sharma, A. 2004. Constraints of Fish Production - A case study in rainfed areas of Uttar Pradesh. Journal of
Interacademica. 8(4). October: 639643.

Sharma, A. and Sharma, Anamika. 2008. Problems faced by the farmers in adoption of improved maize cultivation practices in hills. TJRAR. 8(2): 22-23.

Sharma, Amod. 2011. Economic and Constraints of King Chilli Growers in Dimapur District of Nagaland. Journal of Interacademicia. 15(4): 710-719.

Sharma, Amod. 2012. Inter-state Disparities in Socio-economic Development in North East Region of India. Journal of Agricultural Science. 4(9). September: 236-243.

Sharma, Amod. 2014. Sustainable economic analysis and extent of satisfaction level of King Chilli growers in Nagaland. Agriculture for Sustainable Development. 2(1). June: 188-191.

Sharma, Amod.; Kichu, Yimkumba. and Chaturvedi, B. K. 2016. Economics and Constraints of Pineapple Cultivation in Dimapur District of Nagaland. TJRAR. 16(1). January: 7275.

Sharma, Amod.; Kichu, Yimkumba. and Sharma, Pradeep. Kumar. 2018. Sustainable economic analysis and constraints faced by the pineapple growers in Nagaland. Progressive Agriculture. 18(1). February: 27-33.

Sharma, Rajan., Chauhan, Jitendra., Meena, B. S. and Chauhan, R. S. 2015. Problems Experienced By Farmers and Project Officers in Watershed Management. Indian Research Journal of Extension Education. 15(2\&3): 23-27.

Shuya, Keviu. and Sharma, Amod. 2014. Impact and constraints faced by the borrowers of cooperative bank finance in Nagaland. Economic Affairs. 59(4). October: 561-567.

Shuya, Keviu. and Sharma, Amod. 2018. Problems faced by the Borrowers in 
Utilization and Acquiring of Cooperative Bank Loans in Nagaland. IJED. 14(2). April-June: 52-56.

Tangjang, Avicha. and Sharma, Amod. 2018. Problem faced by the Large Cardamom Growers during production and marketing: A case study of Tirap district of Arunachal Pradesh. IJCMAS. 7(5). May: 2561-2573.

Walling, Imti. and Sharma, Amod. 2015. Impact of SGRY on beneficiaries and non-beneficiaries in Dimapur district of Nagaland. TJRAR. 15(2). August: 90-94.

Walling, Imti.; Sharma, Amod.; Yadav, Mukesh. Kumar.; Rajbhar, Arun, Kumar. and Kalai, Kankabati. 2017. Impact of Agricultural Technology Management Agency on Rural Economy of Nagaland, India. Plant Archiver. 17(2). October: 1511-1516.

\section{How to cite this article:}

Mukesh Kumar Yadav and Amod Sharma. 2019. Livestock Rearing Contribution towards the Beneficiary and non-Beneficiary on Watershed Development Programme in Nagaland. Int.J.Curr.Microbiol.App.Sci. 8(09): 2943-2952. doi: https://doi.org/10.20546/ijcmas.2019.809.338 